\title{
Motivación Intrínseca Laboral y su relación con las variables de personalidad Orientación a la Meta y Tesón
}

\author{
Arturo Solf Zárate
}

Se indagó la relación entre la Motivación Intrínseca Laboral con las variables de personalidad Orientación a la Meta y Tesón (conscientiousness), a fin de reemplazar la variable Necesidad de Crecimiento del Modelo Motivacional propuesto por Hackman y Oldham (1980). Los instrumentos se aplicaron a una muestra de empleados $(N=228)$ de una empresa textil pertenecientes a 60 cargos diferentes. Los resultados mostraron que la Motivación Intrínseca Laboral presentaba una relación positiva con la Orientación a la Meta centrada en el Aprendizaje $(r=0.43 ; p<, 01)$. Finalmente se proporciona sugerencias para una futura investigación sobre el tema, y con fines de aplicación en el ámbito de la administración de los Recursos Humanos.

Motivación Laboral / Motivación Intrínseca / personalidad y trabajo / modelos motivacionales

Intrinsic Motivation at Work and its relationship with the variables Goal Orientation and Tenacity

The relation between the Intrinsic Motivation at Work with the personality variables Goal Orientation and Tenacity (conscientiousness) was investigated, in order to replace the variable Growth Need of the motivational model proposed by Hackman and Oldham (1980). The instruments were applied to a sample of employees $(N=228)$ of a textile company pertaining to 60 different positions. The results showed that the Intrinsic Motivation at Work presented a positive relation with the Goal Orientation centered in the Learning $(r=0.43 ; p<, 01)$. Finally one provides suggestions for future investigation on the subject, and with aims of application in the scope of the administration of the Human Resources.

Work Motivation / Intrinsic Motivation / Personality and Work / Motivational Models 
En los últimos años, en las organizaciones laborales se le está otorgando mayor importancia a la Motivación Intrínseca Laboral, en razón de los cambios que se han suscitado en la sociedad y en la forma de administrar los negocios (Thomas, 2000). En la mayoría de las organizaciones se espera que el trabajador muestre una mayor iniciativa y compromiso con el trabajo para ayudar al éxito organizacional en un medio cada vez más competitivo. Además, se asume que los incentivos extrínsecos (p.ej., dinero, promoción, etcétera) no son suficientes para lograr dicho grado de compromiso de una población de empleados que tiene un mejor nivel educativo y mayor libertad para decidir por sí mismos como consecuencia de la reducción de los niveles de supervisión, y por el uso de la informática en las organizaciones.

Con la finalidad de satisfacer los motivos intrínsecos del empleado, los practicantes y teóricos vinculados a las organizaciones han propuesto modificaciones en el diseño de los puestos y la manera de administrar los Recursos Humanos. Así tenemos el Rediseño y Enriquecimiento Laboral, el Empoderamiento Laboral, Retroinformación $360^{\circ}$, Coaching, etcétera (Jex, 2002). Dichas acciones permitirían al empleado desarrollarse en el trabajo, y como consecuencia se esperaría una mayor satisfacción e identificación con la organización (Kozlowski, Chao, Smith \& Headlung, 1993).
Considerar solo las modificaciones en el diseño de los puestos y/o la forma de administrar los Recursos Humanos no es suficiente para una comprensión integral de la Motivación Intrínseca Laboral, ya que esta es resultado de la interacción de las características del puesto y las peculiaridades de los empleados (Salanova, Hontangas \& Peiró, 1996), destacando en esta última la personalidad y el funcionamiento cognitivo (Jex, 2002).

$\mathrm{Al}$ analizar los estudios sobre la relación entre Motivación Intrínseca y personalidad se encuentra que ha sido objeto de atención la variable Necesidad de Crecimiento propuesta hace unos años por Hackman y Oldham, quienes la definieron como el interés de los sujetos "para el logro personal, aprendizaje, y desarrollar más allá de donde están ahora" (Hackman \& Oldham, 1980: 85). Dichos estudios han proporcionado resultados contradictorios sobre el tipo de relación, los que se supone originados por delimitaciones conceptuales y psicométricas de los instrumentos utilizados (Oldham, 1996).

Los resultados contradictorios sobre la relación entre Motivación Intrínseca Laboral y Necesidad de Crecimiento, motivaron la realización del presente estudio que indaga dicha relación pero utilizando variables de personalidad que actualmente son objeto de investigación, y se supone que presentan similitud conceptual con la Necesidad de 
Crecimiento; además, están mejor definidas y existen instrumentos de medida con adecuadas propiedades psicométricas. Las dimensiones de personalidad seleccionadas para tal fin fueron Orientación a la Meta y Tesón.

La utilidad de la presente investigación es de índole teórica, para contribuir con el modelo de Motivación Laboral sobre las Características del Puesto (Hackman \& Oldham, 1980; Oldham, 1996; Solf, 2004), así como con fines prácticos para la administración de los Recursos Humanos en las organizaciones laborales.

\section{MOTIVACIÓN INTRÍNSECA LABORAL}

La motivación humana es un proceso psicológico vinculado a la activación, dirección y persistencia de la conducta para el logro de determinados objetivos. En el ámbito laboral la motivación ha sido objeto de un amplio análisis, en los que se puede diferenciar dos grupos de motivadores: "extrínsecos" e "intrínsecos". Los "extrínsecos" están fuera del sujeto y pertenecen al ámbito material y social. Los "intrínsecos" se presentan al ejecutar el trabajo y dependen de las características propias del puesto y las del trabajador.

En la investigación sobre la Motivación Intrínseca Laboral podemos identificar dos enfoques: el primero es de contenido, al orientarse a señalar los componentes cognitivos y afectivos que lo conforman. Así Deci y Ryan
(1988) señalan que la autodeterminación y competencia es lo distintivo de la Motivación Intrínseca; para Izard (1977) el interés y excitación; para otros es la felicidad, sorpresa y diversión (Pretty \& Reliman, 1983; Reeve, Cole \& Olson, 1986); para Csokszentmihalyi $(1975,1978)$ es el "flujo" del involucramiento profundo en la tarea (citados en Amabile, Hill, Hennesey \& Tighe, 1994).

El segundo enfoque es de proceso, al indagar la forma como la Motivación Intrínseca Laboral se origina en los individuos. Según esta postura la motivación será una respuesta cognitiva y/o afectiva como consecuencia de la ejecución del trabajo. Como ejemplo de la respuesta cognitiva tenemos a Thomas (2000), quien indica que la Motivación Intrínseca se presentará si el trabajador considera significativo su trabajo, tiene alternativas para ejecutarlo, percibe que le permite adquirir más competencias y es consciente de los avances hacia el objetivo deseado. Con respecto a las consecuencias afectivas destaca el trabajo de Hackman y Oldham (1980), para quienes la Motivación Intrínseca se presentará si los estados afectivos positivos o negativos del sujeto varían según la calidad percibida en la ejecución del puesto, de manera que una persona presentará motivación intrínseca si experimenta sentimientos positivos cuando ejecute bien sus tareas, y sentimientos negativos si rinde mal (Lewicki, 1993). 
En la presente investigación se utilizó el enfoque propuesto por Hackman y Oldham (1980) para la medición de la Motivación Intrínseca Laboral, siendo esta definida como el grado en que los sentimientos del empleado varían en función de la calidad autopercibida de su rendimiento.

\section{Orientación a la Meta}

Las personas que desean lograr una meta específica se pueden diferenciar por los motivos que le inducen a perseguir dichas metas; diferencias que a su vez permitirán efectuar mediciones útiles con fines de diagnóstico y pronóstico. Este es el enfoque que ha perseguido la investigación relacionada con la dimensión Orientación a la Meta, destacando en un comienzo los aportes de Dweck en el ámbito educativo (Dweck \& Elliot, 1983; Dweck \& Leggett, 1988; Dweck \& Sorich, 1999), y luego en las organizaciones laborales a partir de la sugerencia de Button y Mathieu (1996) para ampliar el ámbito de la investigación.

Las razones que sustentan el logro de determinadas metas, a su vez están vinculadas a "pautas características de creencias, atribuciones y afectos del sujeto ante los resultados de éxito o fracaso" (González-Pienda,1996: 167). De acuerdo con esto, se utilizará como definición de Orientación a la Meta el marco cognitivo-perceptual de los sujetos orientados a lograr una meta, sus- tentado por alguna razón, y con modalidades de creencias y conductas que permiten establecer diferencias entre los sujetos.

Dos de los tipos de Orientación a la Meta que se ha investigado más hasta la fecha constituyen la Orientación hacia el Aprendizaje (OM-Aprendizaje) y Orientación hacia el Desempeño (OMDesempeño). En el primer caso los sujetos desean lograr su meta como un medio para adquirir mayor competencia, conseguir un desarrollo personal al aprender nuevas formas y estrategias de desempeño. En el segundo caso a los sujetos les interesa conseguir su meta para demostrar a los demás que son personas capaces y conseguir juicios positivos de aquellos (GonzálezPienda, 1996; Farr, Hofmann \& Ringenbach, 1993; VandeWalle, 1997).

Recientemente se ha establecido una diferenciación en el tipo de OMDesempeño, al identificar dos subtipos: OM-Desempeño-Aproximación y OMDesempeño-Evitación. En el primer caso los sujetos buscan lograr una meta para demostrar a los demás su competencia y obtener juicios positivos de estos. En el segundo caso los sujetos intentan lograr una meta para evitar que los demás los perciban como incompetentes, carentes de capacidad (VandeWalle, 1997).

La investigación actual consideró los tres tipos de Orientación a la Meta descritos anteriormente. 
Según la investigación vinculada a la Orientación a la Meta se ha observado que la OM-Aprendizaje sería más útil para el desempeño de tareas complejas, novedosas o que cambien continuamente, mientras que la OM-Desempeño sería más beneficiosa para la ejecución de tareas simples y rutinarias (VandeWalle, Cron \& Slocum, 2001). En el ámbito de la capacitación los sujetos con mayor OM-Aprendizaje logran un mejor rendimiento y mayor transferencia de lo aprendido (Kozlowski et al., 1993; Ford, Smith, Wiessbein, Gully \& Salas, 1998; Brett \& VandeWalle, 1999; Colquitt \& Simmering, 1998). Además, este tipo de Orientación a la Meta está relacionado con la Autoeficacia, la Necesidad de Logro y el Locus de Control Interno (Phillips \& Gully, 1997).

En relación con la búsqueda de retroinformación los sujetos con alto OM-Aprendizaje efectúan una búsqueda más activa, siendo lo contrario en aquellos con mayor OM-Desempeño (VandeWalle \& Cunmmigs, 1997; VandeWalle et al., 2001).

Al considerar el rendimiento en ciertas actividades, se ha encontrado que en el caso de vendedores con alta OM-Aprendizaje lograban mayores ventas, y no existía relación entre ventas y OM-Desempeño (VandeWalle, Brown, Cron \& Slocum, 1999).

\section{TESÓN}

Actualmente se están utilizando en el ámbito organizacional instrumentos para la evaluación de la personalidad, elaborados en base al modelo de las cinco grandes dimensiones de la personalidad o Big Five (Schneider \& Hough, 1995). Las dimensiones son: Energía o Extraversión, Afabilidad o Agrado, Tesón, Estabilidad Emocional y Apertura a la Experiencia (Caparara, Barbaranelli \& Borgogni, 1995). La dimensión Tesón, tal como se denomina en la prueba de Caprara et al. (1995), también se ha traducido como Conciencia (Laak, 1996), siendo el término conscientiousness el utilizado inicialmente en los test en inglés (Costa \& McCrae, 1992).

De las cinco dimensiones de la personalidad se ha escogido Tesón para el presente trabajo, en razón de que suponemos que coincide conceptualmente con la variable Necesidad de Crecimiento de Hackman y Oldham (1980), pero tiene la ventaja de estar mejor validada y ser motivo de actual investigación. Tesón mide la capacidad de ser perseverante, planificado, responsable e interesado por el logro (Costa \& McCrae, 1992).

La dimensión Tesón "puede ser la variable motivacional-rasgo más importante en el ámbito laboral" (Barrick, Mount \& Strauss, 1993: 721; citado en Colquitt \& Simmering, 1998). Así tenemos que Salgado (1997), basado en 
un metaanálisis de 36 investigaciones realizadas por la Comunidad Europea, concluyó que de las cinco dimensiones sólo Tesón y Estabilidad Emocional eran predictores válidos del desempeño en diversos grupos ocupacionales. Otras investigaciones han demostrado la relación de Tesón con la capacitación laboral (Colquitt, Lepine \& Noe, 2000; Martocchio \& Judge, 1997), el estilo de solución de conflictos (Antonioni, 1998), la Evaluación del Desempeño (Bernandin, Cooke \& Villanova, 2000), la búsqueda de trabajo, continuidad en la organización y asistencia (Judge, Higgins, Thoresen \& Barrica, 1999), la autonomía y autoeficacia (Barrick \& Mount, 1993; Thoms, Moore \& Scout, 1996), y hay evidencia de que los sujetos con alto Tesón viven más tiempo (Friedman et al., 1995; citado en Judge et al., 1999), y presentan menos índice de accidentes al conducir vehículos motorizados (Arthur \& Graciano, 1996).

\section{ReLACIÓn ENTRE MOtivaCIÓN INTRÍNSECA y ORIENTACIÓN a la Meta}

A continuación presentamos algunos resultados de investigaciones vinculadas a la relación entre Motivación Intrínseca y Orientación a la Meta.

Elliot y Harackiewicz (1996) encontraron en un experimento que la OM-Desempeño-Evitación disminuía la Motivación Intrínseca de los sujetos, a diferencia de la OM-Aprendizaje y
OM-Desempeño-Aproximación, que lo incrementaban.

En el estudio de Harackiewicz y Elliot (1993) se analizó el rol moderador de la Motivación de Logro entre la Motivación Intrínseca y Orientación de Meta, concluyendo que la OM-Desempeño aumentó la Motivación Intrínseca en los sujetos con alta Motivación de Logro, mientras que la OM-Aprendizaje realzó el interés intrínseco en aquellos con baja Motivación de Logro. El rol moderador del grado de dificultad de las tareas en la relación entre Motivación Intrínseca y Orientación a la Meta fue investigado por SteeleJohnson, Beuauregard, Hoover y Schmidt (2000), quienes demostraron que en el caso de tareas fáciles la OMDesempeño se vinculaba más con la Motivación Intrínseca, mientras que en las tareas complejas la OM-Aprendizaje estaba más vinculada a la Motivación.

Otra variable moderadora investigada en la relación entre Motivación Intrínseca Laboral y Orientación a la Meta es el tipo de estimulación del contexto, habiéndose encontrado que la Motivación Intrínseca aumenta en los sujetos con alta OM-Aprendizaje cuando el contexto es neutral (no se estimula ninguna Orientación a la Meta), pero en el caso de estimularse el desempeño los sujetos con alta OM-Desempeño presentaban mayor Motivación Intrínseca (Harackiewicz \& Elliot, 1998). 


\section{Relación ENTRE MOtivación INTRÍNSECA Y TESÓN}

No se ha identificado alguna investigación que indague de manera explícita la relación directa entre Motivación Intrínseca y Tesón, aunque algunos trabajos pueden ayudar indirectamente a entender dicha relación. Caligiuri (2000) encontró que Tesón está relacionado con la Evaluación de Desempeño de un grupo de empleados $(\mathrm{N}=143)$ efectuada por sus supervisores. En base a este resultado podemos suponer la presencia, en el nivel del Desempeño Laboral, de la Motivación Intrínseca como uno de los factores que incide (Oldham, 1996).

Otro trabajo es el de Judge et al. (1999), quien resume el aporte de otros investigadores y proporciona resultados que apoyan la relación entre Tesón y ciertos indicadores vinculados a la Motivación, como es el caso de la continuidad y asistencia al trabajo.

\section{Hipótesis de trabajo}

De acuerdo con el análisis bibliográfico efectuado, se establecieron las siguientes hipótesis de trabajo:

- La Motivación Intrínseca Laboral está relacionada positivamente con la OM-Aprendizaje y la OMDesempeño-Aproximación.

- La Motivación Intrínseca Laboral está relacionada negativamente a la OM-Desempeño-Evitación.
- La Motivación Intrínseca Laboral está relacionada positivamente a Tesón.

\section{MÉTOdo}

\section{Sujetos:}

Para el presente trabajo se utilizaron los datos de una investigación de mayor alcance realizada por el autor (Solf, 2004). La población considerada fue una empresa textil ubicada en la ciudad de Lima. La muestra utilizada corresponde a 228 sujetos, que es el 73,55\% de la población total de la empresa, e incluía personal perteneciente a los 60 cargos existentes en la organización.

Para la obtención de la muestra se efectuó un muestreo no probabilísticointencionado (Alarcón, 1991). El 51\% era personal femenino; según la edad el $46 \%$ tenía de 31 a 40 años; de acuerdo con el estado civil el $64 \%$ estaba casado; el $61 \%$ poseía secundaria completa y el $40 \%$ presentaba una antigüedad en el puesto de 6 a 10 años.

\section{Instrumentos}

- Motivación Intrínseca Laboral.- La medición de la Motivación Intrínseca Laboral se efectúo en base a la Escala de Hackman y Oldham incluida en su Job Diagnostic Survey (1980). De las seis afirmaciones originales se concluyó en cuatro a partir de la validación de contenido en 
castellano en base a la opinión de 10 jueces (tres profesores universitarios, cuatro supervisores y tres trabajadores). Para la Escala se obtuvo un Alfa de Cronbach de 0.65.

La escala final utilizada estaba orientada a medir el grado en que el sujeto variaba su estado de ánimo de acuerdo a la calidad autopercibida de su desempeño. Los ítems fueron los siguientes: "Siento una gran satisfacción personal cuando hago bien este trabajo", "Me siento muy mal cuando descubro que he desempeñado mal este trabajo", "Me siento contento cuando realizo bien este trabajo", "Mis sentimientos generalmente no varían cuando desempeño bien este trabajo". Para responder la escala el sujeto tenía que determinar en qué medida estaba de acuerdo con cada afirmación, utilizando para ello una escala tipo Likert que va desde 1 (Total desacuerdo) hasta 6 (Total acuerdo).

- Orientación a la Meta.- Para la medición de la "Orientación a la Meta" se utilizó el Work Domain Goal Orientation Instrument, elaborado por VandeWalle (1997). La validación de contenido en castellano se efectuó en base a 10 jueces (tres profesores universitarios, cuatro supervisores y tres trabajadores). Todos los ítems originales del Instrumento quedaron en la versión utilizada. En relación con la confiabilidad del Instrumento se obtuvo alfas de Cronbach de $0.62,0.64$ y 0.60 para las OM-Aprendizaje, OMDesempeño-Aproximación y OMDesempeño-Evitación, respectivamente.

El total de ítems del Instrumento fueron 13, de los cuales cinco ítems medían OM-Aprendizaje (p.ej., "Me agrada realizar tareas difíciles y desafiantes en el trabajo que me permitan aprender nuevas habilidades"; "A menudo busco oportunidades para desarrollar nuevas habilidades y conocimientos"); cuatro ítems medían OM-DesempeñoAproximación (p.ej., "Busco identificar qué aspectos pueden demostrar a los demás mi capacidad en el trabajo"; "Me alegra saber que otros en el trabajo estén enterados de mi buen desempeño laboral"); y cuatro ítems medían OM-Desempeño-Evitación (p.ej., "Para mí, ocultar una baja capacidad es más importante que aprender una nueva habilidad"; "Me preocupa aceptar una tarea laboral, y que mi desempeño revele baja capacidad"). Para responder el sujeto tenía que decidir en qué medida estaba de acuerdo con cada afirmación, empleando una escala tipo Likert que va desde 1 (Totalmente de acuerdo) a 6 (Totalmente en desacuerdo).

- Tesón.- Para medir esta variable se consideró la escala de Tesón que es una de las cinco dimensiones que forman parte del cuestionario Big 
Five de Caprara et al. (1995), el cual a su vez es una adaptación del italiano al castellano por la editora española TEA.

La escala de Tesón incluye dos subfactores: Escrupulosidad, que mide aspectos relativos a fiabilidad, meticulosidad y amor por el orden; y Perseverancia, que está vinculada a la tenacidad con que se llevan a cabo las tareas y actividades emprendidas, y a cumplir lo prometido. La escala está conformada por 24 ítems, 12 para cada subfactor (p.ej., "Antes de entregar un trabajo, dedico mucho tiempo a revisarlo", "Difícilmente desisto de una actividad que he comenzado"). Los sujetos tenían que indicar el grado de acuerdo con cada afirmación, empleando una escala tipo Likert que va desde 1 (Totalmente en desacuerdo) hasta 6 (Totalmente de acuerdo).

\section{Procedimiento}

Se efectuaron las coordinaciones respectivas con el gerente de Recursos Humanos del centro laboral, quien con los demás jefes planificó la aplicación del instrumento, la cual se llevó a efecto en un salón utilizado con fines de capacitación, durante los meses de abril y mayo del 2002. La aplicación se realizó bajo la responsabilidad de psicólogos, en pequeños grupos de aproximadamente 10 a 15 trabajadores pertenecientes a las diferentes áreas de la empresa. Las respuestas eran anónimas. Los participantes mostraron colaboración al contestar la encuesta.

\section{Resultados}

En primer lugar se presentan, en la tabla 1, las medias aritméticas, desviación estándar y los coeficientes de Kolmogorov-Srmirnov. Estos últimos con la finalidad de detectar en qué medida la distribución de las variables se aproxima a la curva normal teórica.

Tabla 1

Bondad de ajuste de las variables estudiadas a la curva normal teórica

\begin{tabular}{lrrrc}
\hline Variable & $\mathrm{M}$ & $\mathrm{D} . \mathrm{S}$ & $\mathrm{K}-\mathrm{S}-\mathrm{Z}$ & $\mathrm{P}$ a dos colas \\
\hline Motivación Intrínseca & 16.28 & 1.63 & 3.767 & $0.000^{*}$ \\
OM-Aprendizaje & 25.79 & 2.91 & 2.550 & $0.000^{*}$ \\
OM-Des.-Aproximación & 18.71 & 3.11 & 2.070 & $0.000^{*}$ \\
OM-Des.-Evitación & 11.82 & 3.88 & 2.300 & 0.000 * \\
Tesón & 103.59 & 10.52 & 1.070 & 0.202 \\
Escrupulosidad & 49.72 & 7.07 & 1.170 & 0.127 \\
Perseverancia & 53.87 & 5.71 & 1.751 & 0.000 * \\
\hline
\end{tabular}

${ }^{*} p<, 05$ 
Tabla 2

Matriz correlacional de las variables investigadas

\begin{tabular}{|c|c|c|c|c|c|c|c|}
\hline Variable & 1 & 2 & 3 & 4 & 5 & 6 & 7 \\
\hline \multicolumn{8}{|l|}{ 1. Motivación Intrínseca } \\
\hline 2. OM-Aprendizaje & $.43^{* *}$ & & & & & & \\
\hline 3. OM-Des.-Aproximación & .22 & $.36^{\star *}$ & & & & & \\
\hline 4. OM-Des.-Evitación & .07 & -.16 & $.27^{*}$ & & & & \\
\hline 5. Tesón & .13 & $.39^{* *}$ & .05 & $-.28^{*}$ & & & \\
\hline 6. Escrupulosidad & .05 & $.27^{*}$ & .14 & -.03 & $.86^{\star *}$ & & \\
\hline 7. Perseverancia & .17 & $.37^{\star}$ & -.09 & $-.48^{* *}$ & $.78^{\star \star}$ & $.33^{* *}$ & \\
\hline
\end{tabular}

$\mathrm{N}=228$

* $p<, 05$

** $p<, 01$

Según los resultados presentados en la tabla 1, todos los coeficientes K-S y $\mathrm{Z}$ son significativos, excepto Tesón $\mathrm{y}$ Escrupulosidad, siendo así los únicos que se aproximan a una distribución normal. A pesar de que la mayoría de las variables investigadas no presentan una distribución próxima a una curva normal se decidió utilizar un análisis estadístico paramétrico, en consideración al tamaño de la muestra, y además la Correlación de Pearson utilizada puede ser útil a pesar de dicha limitación (Hair, Anderson, Tatham \& Black, 1999).

A continuación, en la Tabla 2 se presenta la matriz de los coeficientes de correlación lineal de Pearson de las variables estudiadas.

De acuerdo con los resultados incluidos en la tabla 2 podemos observar que la Motivación Intrínseca Laboral tiene una relación significativa solo con la OM-Aprendizaje $(\mathrm{r}=0.43$; $\mathrm{p}<, 01)$. Otros resultados por resaltar

son la relación de la OM-Aprendizaje con la OM-Desempeño-Aproximación $(\mathrm{r}=0.36 ; \mathrm{p}<, 01)$ y con Tesón, tanto de manera global $(r=0.39 ; p<, 01)$ como por subfactores, así como la relación negativa entre perseverancia y OMAproximación-Evitación $(\mathrm{r}=-0.48)$.

\section{DISCUSIÓN}

De las relaciones positivas que se esperaba que existieran entre Motivación Intrínseca Laboral y las variables Orientación a la Meta y Tesón solo se encontró entre Motivación Intrínseca y el tipo de Orientación de Meta: OMAprendizaje. A continuación analizamos dicha relación.

Según la investigación sobre la relación entre Motivación Intrínseca y Orientación a la Meta, se ha demostrado que se encontraba una mejor relación cuando se consideraban determinadas variables moderadoras, tal como Motivación de Logro (Harackiewicz \& Elliot, 1993), grado de dificultad de la 
tarea (Steele-Jhonson et al., 2000), estímulo del contexto (Harackiewicz \& Elliot, 1993), el Potencial Motivador del Puesto (Solf, 2004). En el presente estudio, si bien el nivel de la relación es mediano $(r=0.43)$, aún sorprende, por el hecho de que se ha obtenido dicho nivel a pesar de no considerar ninguna variable moderadora en su análisis. En la búsqueda de alguna explicación para dicho resultado, podemos considerar la naturaleza de la conceptualización y medida utilizada para la Motivación Intrínseca Laboral, que se limitaba a respuestas afectivas en función de la calidad percibida de su desempeño. Por otro lado, en las personas interesadas por aprender se presenta normalmente un componente afectivo (Kline \& Saunders, 1993). Lo que estamos afirmando es que la Motivación Intrínseca Laboral, tal como se mide con la propuesta de Hackman y Oldham (1980) y la OM-Aprendizaje poseen un elemento común: la respuesta afectiva.

El análisis efectuado en el párrafo anterior nos lleva a afirmar la necesidad de considerar en el futuro, tanto en la revisión de la literatura científica así como en la ejecución de otros estudios, el tipo de medida de la Motivación Intrínseca Laboral utilizado, el cual puede enfatizar el aspecto afectivo, como es el caso de Hackman y Oldham (1980), o destacar el cognitivo (p.ej., importancia percibida del trabajo, si percibe que le permite adquirir compe- tencias, etcétera) como es el enfoque de Thomas (2000) o Amabile et al. (1994).

Sugerimos que para futuras investigaciones se denomine Respuesta Emocional Contingente (REC) a la reacción afectiva vinculada a la calidad del desempeño, la cual formaría parte importante de la Motivación Intrínseca Laboral además de los componentes cognitivos. De utilizarse dicha denominación serviría para precisar la consideración que se le está otorgando a la respuesta afectiva de la Motivación Intrínseca independiente de lo cognitivo. Además la REC puede ser objeto de un análisis más fino si se incluyen respuestas emocionales vinculadas a otras reacciones, tales como el orgullo por el trabajo, el ensimismamiento o concentración en las tareas, el aburrimiento, etcétera. De esta manera se ampliaría la indagación hacia el actual interés de investigación sobre las emociones en el trabajo (Solf, 2002; León, 2002).

Una posible línea de investigación sobre la Motivación Intrínseca Laboral, podría indagar tanto los aspectos afectivos y cognitivos de la dimensión, para establecer en qué medida ambos o independientemente son predictivos $\mathrm{o}$ están relacionados a otras variables, como el Desempeño Laboral, Orientación a la Meta, etcétera. Realizando una ampliación de esta perspectiva se podría indagar la Motivación Intrínseca y la Extrínseca. De manera que los sujetos podrían tener diferentes niveles 
en ambos tipos de motivación, y cuyos patrones, a su vez, serían objeto de análisis en su relación con otras variables de investigación.

Desde un punto de vista teórico la relación encontrada entre Motivación Intrínseca Laboral y OM-Aprendizaje permite sugerir reemplazar en el modelo motivacional de Hackman y Oldham (1980) la variable Necesidad de Crecimiento por la OM-Aprendizaje. Esto permitiría actualizar el modelo y ubicarlo dentro de los actuales enfoques del Aprendizaje Organizacional y Grupal (Alcover \& Gil, 2002).

El hecho de que la Motivación Intrínseca Laboral no esté relacionada con los dos subtipos de la OMDesempeño puede deberse a que en ambos casos es muy importante el factor social; los sujetos tratan de lograr un objetivo teniendo en consideración a los demás, ya sea para demostrarles que son capaces (OM-DesempeñoAproximación) o evitar opiniones negativas de parte de ellos (OMDesempeño-Evitación). La presencia del factor social requeriría tener en consideración otras características organizacionales, que podrían incrementar, disminuir o anular las relaciones entre Motivación Intrínseca Laboral y OM-Desempeño. Entre dichas características podemos mencionar, por ejemplo, la confianza interpersonal entre trabajadores, la credibilidad de los jefes frente al personal, las propiedades del sistema de retroinformación del desempeño, los criterios que sustentan el prestigio y estatus social, etcétera. Como se puede ver, esta relación parece ser más compleja que la mostrada entre Motivación Intrínseca y $\mathrm{OM}$ Aprendizaje.

Si bien no hay una relación directa entre Motivación Intrínseca y Tesón, se lograría un análisis más fino de considerarse la presencia de variables mediadoras y/o moderadoras. Así, por ejemplo, se puede considerar a la OMAprendizaje como variable moderadora entre Motivación Intrínseca y Tesón. Observando los resultados de la investigación se encuentra que la OMAprendizaje está relacionada positivamente con Motivación Intrínseca Laboral y Tesón, lo cual nos permitiría esperar que habría una relación entre Motivación y Tesón en el caso de presentarse una alta OM-Aprendizaje, sucediendo lo contrario de existir baja OMAprendizaje. De manera similar se puede establecer más hipótesis de investigación incluyendo otras variables, como Autoeficacia, Motivación de Logro, "Locus de Control", Experiencia, Dificultad de tarea, etcétera. Igual razonamiento se puede utilizar para investigar la relación entre Motivación Intrínseca Laboral y Desempeño de los sujetos en sus puestos de trabajo.

Un resultado que va más allá de Motivación y Tesón ya analizado es la relación entre los dos tipos de OMDesempeño con los dos subfactores de Tesón. Así, los sujetos con OM- 
Desempeño-Evitación presentan una relación negativa con Perseverancia $(\mathrm{r}=-0.42)$; esto es, que a mayor Evitación presentarán menos Perseverancia. Mientras que no hay relación entre OM-Desempeño-Aproximación y Perseverancia. Este resultado refuerza la necesidad de considerar dos tipos diferentes de OM-Desempeño, como lo demostró anteriormente VandeWalle (1997).

Desde un punto de vista práctico la relación encontrada entre Motivación Intrínseca y OM-Aprendizaje puede servir en el proceso de evaluación psicológica en el ámbito laboral, como podría ser en el caso de selección de personal, donde un posible indicador de la presencia de OM-Aprendizaje en el evaluado sería el nivel de variabilidad de su estado de ánimo en función de la calidad autopercibida de su desempeño laboral. Otro aspecto práctico que hay que destacar de los resultados es la relación que presenta $\mathrm{OM}$ Aprendizaje con Tesón $(r=0.39)$ y con la OM-Desempeño-Aproximación $(\mathrm{r}=$ 0.36). Esto nos permite recomendar que en la práctica laboral se otorgue la debida importancia a la variable OMAprendizaje al evaluar los Recursos Humanos en función de objetivos establecidos.

En la realización de diagnósticos psicolaborales puede ser útil el resultado de la investigación, de manera que en los instrumentos se incluyan ítems sobre las reacciones afectivas de los trabajadores según su desempeño laboral, lo cual ayudaría a tener una aproximación de los niveles presentes de OM-Aprendizaje en las áreas o grupos laborales. Esto sería complementario a la tradicional indagación sobre Satisfacción Laboral y Clima Organizacional. Por otro lado, también sería de utilidad en el ámbito de la capacitación de personal, para facilitar la designación de personal para que siga cursos que tengan como requisito determinados niveles de OM-Aprendizaje. Finalmente, se podría considerar en los programas de consejería o coaching orientados a lograr que los trabajadores tengan una mejor comprensión de sí mismos y de su conducta, utilizándose para ello la retroinformación dentro de los parámetros de la Orientación de Meta y Motivación Intrínseca.

\section{REFERENCIAS}

Alarcón, R. (1991). Métodos y diseños de investigación del comportamiento. Lima: Universidad Peruana Cayetano Heredia.

Alcover, C. M. \& Gil, F. (2002). Crear conocimiento colectivamente: Aprendizaje organizacional y grupal. Revista de Psicología del Trabajo y de las Organizaciones, 18 (2-3), 259-301.

Amabile, T.; Hill, K.; Hennesey, B. \& Tighe, E. (1994). The work preference inventory: Assessing Iintrinsic and Extrinsic Motivational Orientations. Journal of Personality and Social Psychology, 66, 950-967. 
Antonioni, D. (1998). Relationship between the big five personality factors and conflict management styles. International Journal of Conflict Management, 9, 336-355.

Arthur, W. \& Graciano, W. (1996). The five-factor model, conscientiousness and driving accident involvement. Journal of personality, 64(3), 593-618.

Barrick, M. \& Mount, M. (1993). Autonomy as a moderator of the relationship between the big five personality dimensions and job performance. Journal of Applied Psychology, 78, 111-118.

Bernandin, H.; Cooke, D. \& Villanova, P. (2000). Conscientiousness and agreeableness as predictor of rating leniency. Journal of Applied Psychology, 85, 232-234.

Brett, J. F. \& VandeWalle, D. (1999). Goal Orientation and Goal Content as Predictors of Performance in a Training Program. Journal of Applied Psychology, 84, 863-873.

Button, S. \& Mathieu, J. (1996). Goal Orientation in organizational research: A conceptual and empirical foundation. Organizational Behavior and Human Decision Processes, 67(1), 26-48.

Caligiuri, P. (2000). The big five personality characteristics as predictors of expatriate's desire to terminate the assignment and supervisor-rated performance. Personnel Psychology, 53, 67-88.

Caprara, G.; Barbaranelli, C. \& Borgogni, L. (1995). Cuestionario "Big Five". Madrid: TEA.

Colquitt, J.; Lepine, J. \& Noe, R. (2000). Toward an integrative theory of training motivation: A meta-analytic path analysis of 20 years of research. Journal of Applies Psychology, 85, 678-707.

Colquitt, J. \& Simmering, M. (1998). Conscientiousness, Goal Orientation, and motivation to learn during the learning process: A longitudinal study. Journal of Applied Psychology, 83, 654-665.

Costa, P. T. \& McCrae, R. R. (1992). Revised NEO Personality Inventory. Florida: PAR.

Costa, P. T. (1996). Work and Personality: Use of the NEO-PI-R in Industrial/Organizational Psychology. Applied Psychology: An International Review, 45, 225-241.

Dweck, C. S. \& Elliott, E. S. (1983). Achievement motivation. En: P. Mussen \& E. M. Hetherington (Eds.). Handbook of child psychology. Nueva York: Wiley.

Dweck, C. S. \& Leggett, E. L. (1988). A social-cognitive approach to motivation and personality. Psychological Review, 95, 256-273.

Dweck, C. S. \& Sorich, L. (1999). Mastery-oriented thinking. En: C. R. Snyder (Ed.). Coping. Nueva York: Oxford University Press.

Elliot, A. \& Harackiewicz, J. (1996). Approach an avoidance Achievement Goals and Intrinsic Motivation: A mediational analysis. Journal of Personality and Social Psychology, 70, 461-475.

Farr, J. L.; Hofmann, D. A. \& Ringenbach, K. L. (1993). Goal orientation and action control theory: Implications for industrial and organizational psychology. En: C. L. Cooper \& I. T. Roberston (Eds.). International Review 
of Industrial and Organizational Psychology. Chichester: John Wiley.

Fisher, C. D. (2003). Why do lay people believe that satisfaction and performance are correlated? Possible sources of a commonsense theory. Journal of Organizational Behavior, 24, 753-777.

Ford, J.; Smith, E.; Weissbein, D.; Gully, S. \& Salas, E. (1998). Relationships of goal orientation, metacognitive activity and practice strategies with learning outcomes and transfer. Journal of Applied Psychology, 83, 218-233.

Goldberg, C. B. \& Waldman, D. A. (2000). Modeling employee absenteeism: Testing alternative measures and mediated effects based on Job Satisfaction. Journal of Organizational Behavior, 21, 665-676.

González-Pienda, J. A. (1996). El estudiante: Variables personales. En: J. Beltrán \& A. Génova (Eds.). Psicología instruccional. Madrid: Síntesis.

Hackman, J. R. (2003). Learning more by crossing levels: Evidence from airplanes, hospitals, and orchestras. Journal of Organizational Behavior, 24, 905-922.

Hair, J.; Anderson, R.; Tatham, R. \& Black, W. (1999). Análisis multivariante. Madrid: Prentice Hall.

Harackiewicz, J. \& Elliot, A. (1993). Achievement goals and intrinsic motivation. Journal of Personality and Social Psychology, 65, 904-915.

Harackiewicz, J. \& Elliot, A. (1998). The joint effects of target and purpose goal on intrinsic motivation: A mediational analysis. Personality and Social Psychology Bulletin, 24, 675-689.
Hackman, J. R., \& Oldham, G. R. (1980). Work redesign. Readings, MA.: Addison-Wesley.

Jex, S. M. (2002). Organizational psychology. A scientist-practitioner approach. Nueva York: John Wiley \& Sons.

Judge, T.; Higgins, C.; Thoresen, C. \& Barrick, M. (1999). The Big Five personality traits, general mental ability, and career success across the life span. Personnel Psychology, 52, 621-652.

Kanfer, R., (1990). Motivation theory and industrial and organizational psychology. En: M. D. Dunnette \& L. M. Hough, (Eds.). Handbook of Industrial \& Organizational Psychology, (Vol. 1, 2. ${ }^{a}$ edición). California: Consulting Psychologists Press.

Kline, P. \& Saunders, B. (1993). Ten steps to a learning organization. Virginia: Great Ocean.

Kozlowski, S.; Chao, G.; Smith, E. \& Hedlund, J. (1993). Organizational downsizing: Strategies, interventions and research implications. En: C. L. Cooper \& I. T. Robertson (Eds.). International Review of Industrial and Organizational Psychology. Chichester: John Wiley \& Sons.

Laak, J. (1996). Las cinco grandes dimensiones de la personalidad. Revista de Psicología, XIV(2).

León, R. (2002). Un estudio acerca de la envidia en los centros laborales en el Perú. Persona, 5, 147-165.

Lewicki, R. (1993). Desarrollo organizacional. Guía de capacitación. México: Limusa. (Edición en inglés: 1988)

Martocchio, J. \& Judge, T. (1997). Relationship between conscientiousness and 
learning in employee training: Mediating influences of self-deception and self-efficacy. Journal of Applied Psychology, 82, 764-773.

Oldham, G. R. (1996). Job Design. En: C. L. Cooper \& I. T. Roberston (Eds.). International Review of Industrial and Organizational Psychology. Chichester: John Wiley.

Phillips, J. M. \& Gully, S. M. (1997). Role of goal orientation, ability, meed for achievement, and Locus of Control in the Self-Efficacy and Goal-Setting Process. Journal of Applied Psychology, 82, 792-802.

Salanova, M.; Hontangas, P. \& Peiró J. (1996). Motivación Laboral. En: J. Ma . Peiró y F. Prieto (Eds.). Tratado de psicología del trabajo, vol. 1. Madrid: Síntesis.

Salgado, J. F. (1997). The five factor model of personality and job performance en the European community. Journal of Applied Psychology, 82, 30-43.

Schneider, R. J. \& Hough, L. M. (1995). Personality and industrial/organizational psychology. En: C. L. Cooper \& I. T. Roberston (Eds.). International Review of Industrial and Organizational Psychology. Chichester: John Wiley.

Solf, A. G. (2002). Las emociones en el trabajo. Revista Peruana de Psicología. Número monográfico dedicado a Inteligencia Emocional, 12(7), 49-53. Colegio de Psicólogos del Perú.

Solf, A. G. (2004). Tesón y Orientación de Meta como elementos del modelo motivacional de Hackman y Oldham. Tesis de maestría no publicada. Lima: Universidad Nacional Mayor de San Marcos.

Steele-Johnson, D.; Beauregard, R. S.; Hoover, P. B. \& Schmidt, A. M. (2000). Goal Orientation and task demand effects on motivation, affect, and performance. Journal of Applied Psychology, 85, 724-738.

Thomas, K. W. (2000). Intrinsic motivation at work. Building energy \& commitment. San Francisco: Berrett-Koehler.

Thoms, P.; Moore, K. S. \& Scott, K. S. (1996). The relationship between selfefficacy for participating in self-managed work groups and the Big Five personality dimensions. Journal of Organizational Behavior, 17, 349-362.

VandeWalle, D., (1997). Development and validation of a work domain Goal Orientation instrument. Educational and Psychological Measurement, 57(6), 995-1015.

VadeWalle, D.; Brown, S.; Cron, W. \& Slocum, J. (1999). The influence of Goal Orientation and Self-Regulation tactics on sales performance: A longitudinal field test. Journal of Applied Psychology, 84, 249-259.

VandeWalle, D.; Cron, W. \& Slocum, J. (2001). The role of Goal Orientation following performance feedback. Journal of Applied Psychology, 86, 629-640.

VandeWalle, D. \& Cummings, L. L. (1997). A test of the influence of Goal Orientation on the feedback-seeking process. Journal of Applied Psychology, 82, 390-400. 